\title{
THE IMPORTANCE OF SOFT SKILLS AND IT PROJECT MANAGERS' PERSONALITY TYPE
}

\begin{abstract}
Information technology (IT) is a strategic element in business as it is fundamental to create value to organizations. To be efficient, project managers need to develop other competencies besides their technical skills ("hard skills"). This paper aims to show the importance of soft skills in the development of IT project managers' competencies by answering the following questions (1) "which is the impact of "soft skills" on IT projects management?" and (2) "the personality type of the project manager can influence the development of his/her soft skills?". To answer these questions, we conducted an exploratory and qualitative research. To collect data, we interviewed IT project managers and applied the MBTI test to identify their personality type. As we crossed the results of MBTI tests and content of the interviews, we noticed that some professionals have a personality type that might hinder them from developing some soft skills. Another important finding is that leadership and communication are the most important soft skills in project management according to IT project managers. This research contributes to the academia as it indicates opportunities for further studies on the relationship of personality type and competence development. A managerial implication of research results is that managers and leaders can use personality tests such as MBTI to prevent relationship conflicts in teams as well as to design training programs that best suit their subordinates' personality traits.
\end{abstract}

KEY WORDS: MBTI; Soft Skills; Project Manager Competencies; Information Technology; Competence Development

Cíntia Araújo ${ }^{1}$

Cristiane Drebes Pedron ${ }^{2}$

\footnotetext{
${ }^{1}$ Universidade Nove de Julho - UNINOVE, Brasil

E-mail: cintyaraujo@gmail.com

${ }^{2}$ Universidade Nove de Julho - UNINOVE, Brasil

E-mail: cdpedron@ gmail.com
} 


\section{A IMPORTÂNCIA DOS SOFT SKILLS E O TIPO DE PERSONALIDADE DOS GERENTES DE PROJETOS DE TI}

\section{RESUMO}

A Tecnologia da Informação (TI) é elemento estratégico nos negócios e da criação de valor para as organizações. Pesquisas indicam que para obter sucesso, os gerentes de projetos precisam desenvolver outras competências além das competências técnicas ("hard skills"). Esta pesquisa tem por objetivo mostrar a importância dos soft skills no desenvolvimento das competências dos gerentes de projetos de TI ao responder às seguintes questões (1) "qual o impacto dos soft skills no gerenciamento de projetos de TI?" e (2) "o tipo de personalidade do gerente de projetos pode influenciar no desenvolvimento de seus soft skills?". Para responder a estas perguntas nós conduzimos uma pesquisa exploratória e qualitativa. Para coletar dados, nós entrevistamos gerentes de projetos de TI e aplicamos o teste MBTI para identificar o tipo de personalidade dos entrevistados. Ao cruzar os resultados do teste MBTI e o conteúdo das entrevistas, nós observamos que alguns profissionais têm um tipo de personalidade que pode dificultar o desenvolvimento de soft skills. Outro resultado importante é que liderança e comunicação são os soft skills mais essenciais no gerenciamento de projetos na perspectiva dos gerentes de projetos de TI. Esta pesquisa contribui para a academia ao mostrar oportunidades para mais estudos sobre a relação do tipo de personalidade e desenvolvimento de competências. Dentre as implicações gerenciais está o fato de que gerentes e lideres podem usar testes de personalidade como o MBTI para prevenir conflitos em equipes e também para elaborar programas de treinamento mais adequados às características de personalidade de seus subordinados.

PALAVRAS-CHAVE: MBTI; Soft Skills; Competências do Gerente de Projetos; Tecnologia da Informação; Desenvolvimento de Competências

\section{INTRODUÇÃOO}

A Tecnologia da Informação (TI) é elemento estratégico da execução dos negócios e da criação de valor nas organizações (Assis, 2011). A importância de TI nas organizações é refletida no crescente investimento feitos na área a fim de aumentar produtividade e melhorar qualidade dos serviços de TI. Em contrapartida, as organizações exigem o uso responsável e ágil dos recursos investidos em TI, exercendo forte pressão para redução custos (Assis, 2011).

Apesar dos esforços em potencializar os serviços de TI nas organizações, o índice de fracassos em projetos de TI é alto. No mundo corporativo é sabido que grande parte dos grandes projetos de TI é entregue em atraso e com estouro de orçamento (Sumner, Bock, \& Giamartino, 2006). Um estudo realizado pelo Standish Group reportou que 34\% dos projetos foram entregues com atraso ou acima do orçamento; $31 \%$ foram cancelados, modificados ou reduzidos. (Sumner et al., 2006). Um estudo de empresas que implantaram sistemas de ERP (Enterprise Resource Planning), Sistemas Integrados de Gestão Empresarial (SIGE), reportou que 55,5\% destas empresas ultrapassaram o orçamento planejado para implantação dos sistemas de ERP (Sumner et al., 2006). A pesquisa do Standish Group apontou também que os fatores impactantes tanto no sucesso como no fracasso dos projetos de TI eram relacionados ao grau de envolvimento do usuário, suporte da gerência e definição dos requerimentos (Rabechini Jr., 2001). 
Wateridge (Wateridge, 1997) argumenta que dentre as principais causas do fracasso de projetos de TI estão: (1) Má definição dos objetivos antes do início dos projetos; (2) Falta de planejamento e controle; e (3) Falta de envolvimento do usuário no projeto.

A partir das pesquisas realizadas por Standish Group e por Wateridge conclui-se que fatores impactantes no sucesso e no fracasso de projetos de TI são pertinentes ao gerenciamento de projetos. Estes resultados evidenciam que o gerente de projetos de TI necessita possuir competências diversas além das competências técnicas ("hard skills"). Rabechini (2001) cita que o gerente de projetos exerce papéis fundamentais, papéis estes fora dos aspectos técnicos como: planejador, organizador, administrador de interfaces, articulador de acordos, administrador de tecnologia, administrador de equipes/pessoas, formulador de métodos e implementador (Maximiano, 1986 como citado em Rabechini, 2001).

Sumner et al. (2006) enfatizam que "soft skills" como habilidade de lidar com pessoas e capacidade de comunicação influenciam diretamente o estilo de gerenciamento do líder de projetos. Estudos comprovaram que profissionais de TI são carentes no desenvolvimento de soft skills. Ao aplicar o teste do MBTI (Myers-Briggs Type Indicator) em profissionais de TI Lyons (1985 como citado em (Sumner et al., 2006) constatou que apenas 19\% tomavam decisões baseados em valores pessoais ou sentimentos e que $67 \%$ eram introvertidos.

Este trabalho tem por objetivo mostrar a importância dos soft skills no desenvolvimento das competências dos gerentes de projetos de TI ao responder às seguintes questões (1) "qual o impacto dos soft skills no gerenciamento de projetos de TI?" e (2) "o tipo de personalidade do gerente de projetos pode influenciar no desenvolvimento de seus soft skills?".

A pesquisa teve uma abordagem qualitativa exploratória, realizada a partir da aplicação do teste do MBTI, entrevistas semi-estruradas e um questionário eletrônico aplicados em profissionais de TI de empresas de diferentes setores de negócio.

Os resultados indicam que das sete competências mais valorizadas pelos respondentes, seis eram relacionadas a soft skills. Segundo os participantes, liderança e comunicação são "skills fundamentais" no gerenciamento de projetos de TI. Outro resultado interessante o tipo de personalidade de alguns profissionais pode limitá-los no desenvolvimento de competências que eles mesmos consideram como fundamentais.

A contribuição desta pesquisa é mostrar oportunidades para estudos futuros sobre (1) a relação entre o tipo de personalidades e o desenvolvimento de habilidades interpessoais e comportamentais dos profissionais de TI e sobre (2) soluções de questões críticas que têm afetado as relações sociais e de comunicação no gerenciamento de projetos de TI (Müller, 2003; Sumner et al., 2006; Wateridge, 1997). Esta pesquisa também é útil como ferramenta para os gestores de TI na potencialização das competências e das habilidades de equipes.

$\mathrm{O}$ artigo apresenta a seguinte estrutura: no referencial teórico apresentamos o conceito de soft skills e sua relação com gerenciamento de projetos, a definição do framework do MBTI e abordagem no MBTI na literatura de gerenciamento de projetos. Na metodologia, temos a descrição do método de pesquisa; na seção seguinte temos de análise de resultados dos testes MBTI, das entrevistas e do survey; e por último, temos a conclusão da pesquisa e as considerações finais.

\section{REFERENCIAL TEÓRICO}

A literatura de gerenciamento de projetos dava mais ênfase à eficiência que a fatores comportamentais e interpessoais (Turner \& Müller, 2005). Porém, as capacidades necessárias para um gerente de projetos eficiente e eficaz não são as mesmas para os profissionais do século XXI (Kerzner, 2009). Antes, o gerenciamento de projetos era feito por engenheiros uma vez que o papel do gerente de projetos era a tomar decisões técnicas. Com o aumento da 
complexidade dos projetos, o gerente de projetos passou de gerente "técnico" para gerente "de negócios" (Kerzner, 2009).

\section{Conceito de competência, habilidade, capacidade e skills}

No âmbito da educação e da psicologia habilidade é definida como "o poder para executar o que se designa de ato responsável. Este poder pode ser potencial, real, inato ou adquirido." (Manfredi, 1998, p. 13) Em gerenciamento de projetos, habilidade é o poder mental, físico, financeiro ou legal para desempenhar uma determinada tarefa (PMI, 2007).

$\mathrm{Na}$ língua inglesa, capacidade é traduzida como capability e capacity. Capacity é a habilidade potencial; capability é a habilidade que depende de treinamento ou exercício (Manfredi, 1998). Segundo Manfredi (1998), existem dois tipos de capacidade: a inata e as adquiridas (resultantes de treinamento ou exercício). Skills (ou competências), por sua vez, expressam os níveis de eficiência de um indivíduo na execução de uma determinada capacidade adquirida (capability) (Manfredi, 1998).

O termo "competência" é usado para assinalar que um indivíduo está qualificado para realizar um determinado trabalho (Fleury \& Fleury, 2001). O conceito de competência é:

"um saber agir e responsável e reconhecido, que implica mobilizar, integrar, transferir

conhecimentos, recursos e habilidades, que agreguem valor econômico à

organização e valor social ao indivíduo" (Fleury \& Fleury, 2001, p. 188).

Em gerenciamento de projetos, competência é dividida em três tipos: individuais, de equipe e da empresa (Frame, 1999 como citado em Rabechini \& Carvalho, 2003). As competências individuais se referem às aptidões e capacidade dos indivíduos na solução de problemas; as de equipe, com a capacidade do grupo para resolução de problemas complexos num cenário multidisciplinar; e as de empresa, à capacidade da organização em criar um ambiente de trabalho que possibilite os indivíduos e equipes executar suas funções dentro dos projetos (Rabechini \& Carvalho, 2003).

\section{Gerenciamento de projetos e os soft skills}

À medida que a atividade de gerenciamento de projetos foi amadurecendo, o papel do gerente de projetos foi se transformando. A exigência da expertise técnica passou a ser dos gerentes funcionais (Kerzner, 2009). A figura 1 mostra a mudança da distribuição das habilidades necessárias a um gerente de projetos entre 1985 e 2008: 
Figura 1: Distribuição das habilidades necessárias para o gerente de projetos entre 1985 e 2008

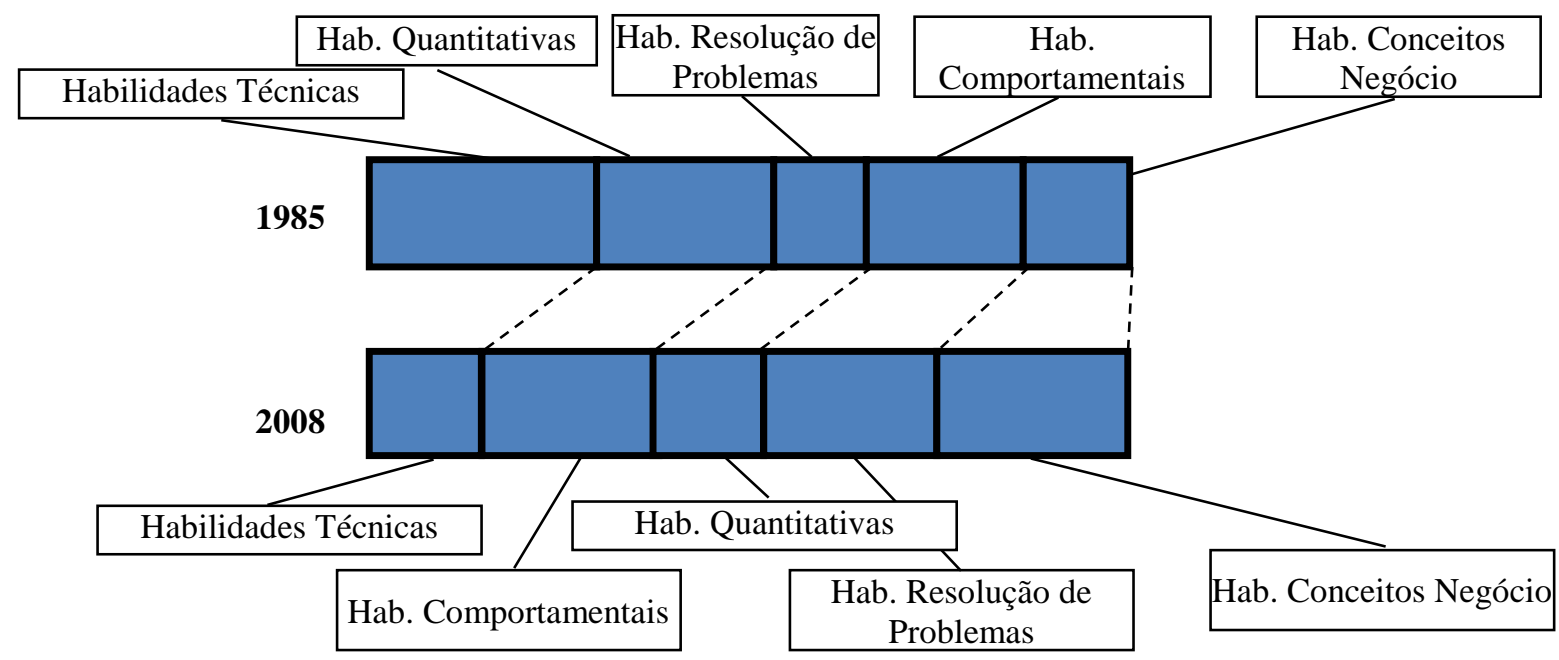

Fonte: Adaptado de (Kerzner, 2009)

A perspectiva de Kerzner (2009) sobre as competências necessárias aos gerentes de projetos do século XXI remete à importância dos soft skills no gerenciamento de projetos. Soft skills são competências comportamentais interpessoais e intrapessoais (Rao, 2012) "que incluem capacidades tais como habilidades de comunicação, inteligência emocional, resolução de conflitos, negociação, influência, construção de equipe e facilitação de grupos." (PMI, 2013). 2012).

Por outro lado, hard skills abrangem conhecimento e competências técnicas (Rao,

A abordagem dos soft skills no gerenciamento de projetos é um tema ainda novo (Rao, 2012) e a literatura de gestão de projetos dá mais ênfase aos hard skills (Pant \& Baroudi, 2008). Todavia, há várias pesquisas que destacam a importância dos soft skills para garantia de bons resultados na gestão de projetos (Stevenson \& Starkweather, 2010; Wateridge, 1997, 1998). Belzer (2001) argumenta que os soft skills são "o elo perdido" no gerenciamento de projetos e crucial para obter sucesso do projeto. E, no gerenciamento de projetos em TI, vemos a mesma tendência: pesquisas e estudos publicados têm comprovado que os soft skills têm impacto direto no resultado de projetos de TI (Stevenson \& Starkweather, 2010; Sumner et al., 2006; Wateridge, 1997, 1998).

A capacitação de gerentes de projetos no âmbito dos soft skills pode ser complexa, uma vez que ao contrário das competências de hard skills, as competências de soft skills são difíceis de quantificar. Porém, gerentes de projetos podem adquirir e aprimorar suas habilidades intrapessoais e interpessoais através de treinamento, observação, leitura e experiência (Rao, 2012; Wateridge, 1997).

\section{Myers-Briggs Type Indicator (MBTI)}

Testes de personalidade têm sido amplamente usados pelas organizações para aprimorar as competências dos indivíduos e potencializar o trabalho em equipe (Rajapogal, 2008). Estimou-se que aproximadamente $\$ 100$ milhões foram investidos ao ano por empresas, na 
realização de teste de personalidade para desenvolvimento de carreira, construção de equipes, e desenvolvimento de gerentes e executivos (Zemke, 1992).

Dentre os testes de personalidade, o MBTI (Myers-Briggs Type Indicator) é um dos mais populares. Gardner et al. (Rajapogal, 2008) reporta que três milhões de testes MBTI são aplicados por ano nos Estados Unidos. O MBTI geralmente é aplicado como ferramenta de auxílio em treinamento de funcionários, identificação de tipos de personalidade de gerentes e no aprimoramento do uso das competências dos indivíduos (Dolfi \& Andrews, 2007; Rajapogal, 2008).

O MBTI foi desenvolvido por Katherine Briggs e por sua filha Isabel Myers nos anos 40. Elas desenvolverem o modelo de MBTI com base na teoria de tipos psicológicos de Carl Jung (Dolfi \& Andrews, 2007). O conceito principal do MBTI é o tipo psicológico. De acordo com este conceito, os indivíduos têm preferências no modo de agir e pensar. O MBTI dividiu as preferências dos indivíduos em quatro pares opostos chamados dicotomias (dimensões). A figura 2 apresenta o relacionamento das quatro dicotomias. Da combinação das quatro dicotomias derivam os dezesseis tipos de personalidade, por exemplo: ESTJ (Extroversion, Sensing, Thinking, Judging).

Figura 2: Relação das dicotomias do MBTI

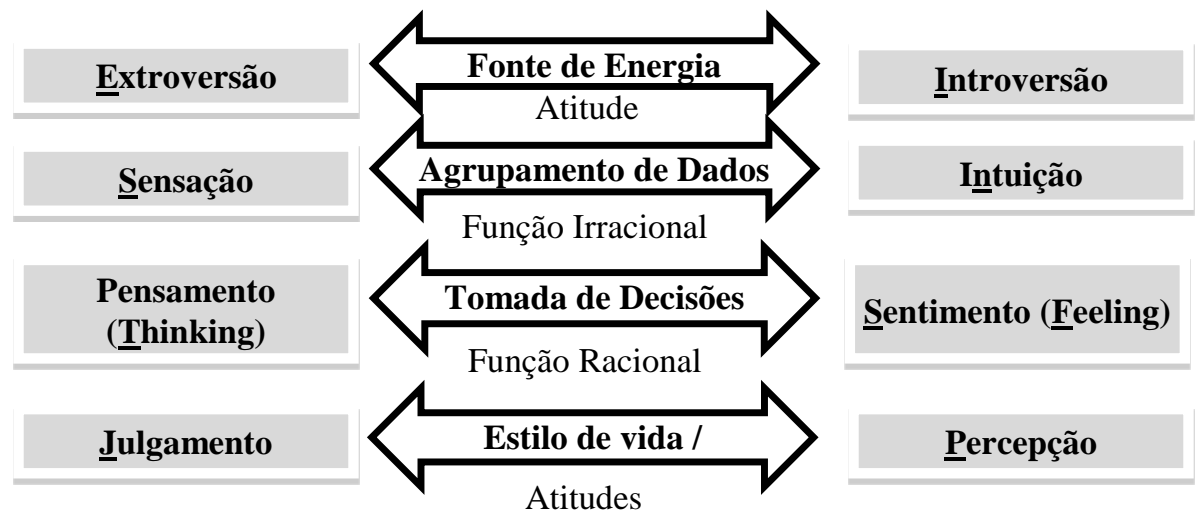

Fonte: Adaptação (Smith, 2001)

A tabela 1 apresenta a síntese das descrições dos dezesseis tipos do MBTI. 
Tabela 1: Síntese da descrição dos tipos de personalidade do MBTI

\begin{tabular}{|c|c|}
\hline Dicotomia & Descrição \\
\hline E ou I & $\begin{array}{l}\text { A escala de extroversão (E) até introversão (I) representa uma medida contínua de como a } \\
\text { pessoa prefere interagir com o meio ambiente. Os mais próximos de (E) direcionam seu foco } \\
\text { para o mundo do outro e estão mais disponíveis para buscar interação a partir de } \\
\text { relacionamento social. São inclinados à comunicação oral mais do que a comunicação escrita. } \\
\text { Os que se orientam mais por I, ao contrário, buscam uma menor interação e intentam resolver } \\
\text { suas necessidades a partir de suas próprias buscas mais "solitárias". }\end{array}$ \\
\hline S ou $N$ & $\begin{array}{l}\text { A escala de sensibilidade (S) até intuição (N) traduz uma medida de como o indivíduo prefere } \\
\text { ou atua para perceber situações ou obter informações. Uma pessoa no nível mais forte de (S) } \\
\text { tende a confiar mais nos sentidos da visão, audição, olfato, paladar e tato, para tratar } \\
\text { informações do meio ambiente. Estes privilegiam maior poder de observação da realidade e } \\
\text { dos fatos e possuem boa memória para detalhes e são práticos. Aqueles que utilizam mais (N) } \\
\text { preferem verificar "o que está por trás" das circunstâncias dadas e percebidas. }\end{array}$ \\
\hline T ou $F$ & $\begin{array}{l}\text { A escala de pensamento }(\mathrm{T}) \text { até sentimento }(\mathrm{F}) \text { indica uma medida de como as pessoas } \\
\text { preferem construir suas conclusões. Os mais inclinados para }(\mathrm{T}) \text { adotam um postura de } \\
\text { racionalidade observacional e intentam fazer uma escolha impessoal baseada em suas } \\
\text { cogitações racionais. Os inclinados ao (T) produzem uma ordenação de fatos e idéias } \\
\text { considerando uma perspectiva de seqüência lógica, com o objetivo de visualizar relações de } \\
\text { causa e efeito. Já os m ais inclinados ao sentimento (F) preferem tomar decisões baseadas em } \\
\text { valores "humanos" e não em "técnica" ou pura "lógica". }\end{array}$ \\
\hline P ou J & $\begin{array}{l}\text { As preferências de personalidade finais apontam para a maneira como o indivíduo negocia } \\
\text { com o mundo exterior. Alguém identificado como utilizando o julgamento (J) sugere uma } \\
\text { pessoa que prefere atuar no processo predominantemente pelo julgamento (pensamento ou } \\
\text { sentimento) para lidar com o mundo exterior. Como resultado, alguém (J) tende a viver dentro } \\
\text { um planejamento e decidir seu modo e ordem, desejando regular e controlar a vida. Uma } \\
\text { pessoa adepta da percepção (P) (sensibilidade ou intuição) prefere esta forma para se } \\
\text { relacionar com o mundo exterior. Uma pessoa mais próxima da (P) é consequentemente mais } \\
\text { flexível e espontânea, desejando entender a vida e sua adaptação a esta. }\end{array}$ \\
\hline
\end{tabular}

Fonte: (Ribeiro Filho, Lopes, Pederneiras, Almeira, \& Ribeiro, 2010, p. 54)

Encontra-se na literatura de gerenciamento de projetos vários os estudos que avaliam a relação entre os tipos de personalidade do MBTI com as competências de gerenciamento de projetos. Diversos autores têm desenvolvido modelos para aprimoramento das competências de gerenciamento de projetos a partir de cruzamento dos tipos de personalidade do MBTI com o as competências relacionadas ao gerenciamento de projetos (Shenhar \& Wideman, 2002; Smith, 2001; Wideman, 1998, 2008).

\section{METODOLOGIA}

Este trabalho tem por objetivo mostrar a importância dos soft skills no desenvolvimento das competências dos gerentes de projetos de TI. Considerando o objetivo do trabalho, a limitação de amostras e o curto prazo para conclusão do trabalho foi realizada uma pesquisa exploratória. A pesquisa exploratória foi adotada porque permite maior flexibilidade no planejamento (Gil, 2002) e também investigar um objeto ou fenômeno com poucas informações (Dalfovo, Lana, \& Silveira, 2008). 
O levantamento (survey) foi o método de pesquisa adotado uma vez que o intuito dos pesquisadores era a de obter o ponto de vista de profissionais que atuam na área de TI, enriquecendo a pesquisa com insights e perspectivas de pessoas que vivenciam as questões de gestão de projetos de TI. No decorrer da pesquisa, constatou-se que o método propiciou uma ótima oportunidade para aplicação de um sistema teórico a situações do cotidiano destes profissionais e desenvolvimento de hipóteses para pesquisas futuras (Martins \& Theóphilo, 2009).

Para coleta de dados foram aplicadas duas técnicas, entrevista e questionário eletrônico. Foram realizadas quatro entrevistas semi-estruturadas com profissionais de TI. As entrevistas foram organizadas utilizando-se a mesma lista de perguntas do questionário eletrônico. A lista de perguntas encontra-se no apêndice A deste artigo.

A elaboração do questionário foi realizada em duas fases: (1) Pesquisa literatura dos soft skills importantes para gerentes de projetos. A partir desta pesquisa, dois trabalhos foram selecionados para servirem de base do questionário: Stevenson \& Starkweather (2010) e Müller \& Turner, (2007). (A tabela 2 apresenta a lista de competências e habilidades contidas no questionário); (2) Validação de face por dois dos profissionais de TI, aplicação do instrumento e comentários sobre o mesmo.

O questionário eletrônico foi desenvolvido com ferramenta do Google Docs ${ }^{\circledR}$ e foi enviado para trinta profissionais de TI, colaboradores de empresas de diferentes setores de negócios. Dos trinta questionários enviados, quatorze foram respondidos. Neste questionário, os profissionais foram inquiridos sobre o grau de importância que eles davam para uma lista de competência e habilidades para gerentes de projetos de TI. A primeira metade das habilidades foi baseada no trabalho de Stevenson \& Starkweather (2010) e; a segunda, em Müller \& Turner, (2007). Para mensuração quantitativa das variáveis qualitativas do questionário aplicado (Martins \& Theóphilo, 2009)foi aplicada a mesma escala Likert de 1 (extremamente nãoimportante) a 5 (extremamente importante) da pesquisa de (Müller \& Turner, 2007).

Tabela 2: Descrição das competências e habilidades usadas no survey

\begin{tabular}{|l|l|}
\hline $\begin{array}{c}\text { Habilidades e } \\
\text { competências }\end{array}$ & \multicolumn{1}{|c|}{ Descrição } \\
\hline Motivação & $\begin{array}{l}\text { Ter energia para alcançar resultados e impactar as pessoas ao redor. Saber equilibrar os } \\
\text { objetivos de curto e longo prazo e perseverar mesmo em meio à rejeição e } \\
\text { questionamentos (Dulewicz \& Higgs, 2005). }\end{array}$ \\
\hline Discernimento & $\begin{array}{l}\text { Demonstrar comprometimento com o objetivo mesmo em face a obstáculos, ter } \\
\text { coerência nos atos e palavras e ser comprimetido com a ética (Dulewicz \& Higgs, 2005). }\end{array}$ \\
\hline Influência & $\begin{array}{l}\text { Persuadir outros a mudar de opinião; ter habilidade de convencer outros a ouvir o seu } \\
\text { ponto de vista (Dulewicz \& Higgs, 2005). }\end{array}$ \\
\hline Autoconsciência & $\begin{array}{l}\text { Ter consciência de seus próprios sentimentos e saber controlá-los. Saber controlar a } \\
\text { influência dos seus sentimentos no ambiente de trabalho (Dulewicz \& Higgs, 2005). }\end{array}$ \\
\hline $\begin{array}{l}\text { Resiliência } \\
\text { emocional }\end{array}$ & $\begin{array}{l}\text { Executar suas funções consistentemente mesmo sob pressão e adaptar seu } \\
\text { comportamento de acordo com as circunstãncias. Ponderar as necessidades de uma } \\
\text { determinada situação em realação às necessidades das pessoas envolvidas (Dulewicz \& } \\
\text { Higgs, 2005). }\end{array}$ \\
\hline Intuição & $\begin{array}{l}\text { Tomar decisões claras e objetivas com base em informações ambíguas e incompletas, a } \\
\text { partir de sua capacidade emocional e racional e de percepção intuitiva (Dulewicz \& } \\
\text { Higgs, 2005). }\end{array}$ \\
\hline
\end{tabular}




\begin{tabular}{|c|c|}
\hline $\begin{array}{l}\text { Habilidades e } \\
\text { competências }\end{array}$ & Descrição \\
\hline $\begin{array}{l}\text { Gerenciar } \\
\text { recursos }\end{array}$ & $\begin{array}{l}\text { Planejar, coordenar e organizar recursos com eficiência e eficácia; estabelecer objetivos } \\
\text { claros; estabelecer tarefas claras e objetivas a partir de planos de longo prazo; monitorar } \\
\text { o desempenho da equipe regularmente e fornecer feedback quando necessário } \\
\text { (Dulewicz \& Higgs, 2005). }\end{array}$ \\
\hline $\begin{array}{l}\text { Habilidade de } \\
\text { persuadir }\end{array}$ & $\begin{array}{l}\text { Saber comunicar com entusiasmo a fim de obter engajamento das pessoas e ganhar } \\
\text { apoio para atingir seus objetivos; dar instruções claras à equipe; ter estilo de } \\
\text { comunicação que inspira a equipe; mostrar à equipe através da comunicação que é um } \\
\text { líder próximo e de fácil acesso (Dulewicz \& Higgs, 2005). }\end{array}$ \\
\hline $\begin{array}{l}\text { Desenvolver } \\
\text { recursos }\end{array}$ & $\begin{array}{l}\text { Acreditar no potencial de seus recursos e encorajá-los a alcançar seu potencial; } \\
\text { desenvolver as competências de seus recursos e fornecer feedback quando necessário } \\
\text { (Dulewicz \& Higgs, 2005). }\end{array}$ \\
\hline Delegar & $\begin{array}{l}\text { Dar autonomia à equipe e enconrajá-la a assumir funções mais desafiadoras; encorajar } \\
\text { os membros da equipe a resolver problemas, ter idéias inovadoras e desenvolver uma } \\
\text { visão mais ampla (Dulewicz \& Higgs, 2005). }\end{array}$ \\
\hline $\begin{array}{l}\text { Habilidade de } \\
\text { realização }\end{array}$ & $\begin{array}{l}\text { Tomar decisões arriscadas com objetivo de obter vantagens, levando em considerando } \\
\text { a estratégia do negócio e o impacto no sucesso do empreendimento; ter determinação } \\
\text { em alcançar resultados e implementar decisões (Dulewicz \& Higgs, 2005). }\end{array}$ \\
\hline $\begin{array}{l}\text { Perspectiva } \\
\text { estratégica }\end{array}$ & $\begin{array}{l}\text { Ter visão ampla das questões e de suas implicações; ter noção do impacto de } \\
\text { relacionamentos em questões futuras; ter sensibilidade para avaliar o impacto das } \\
\text { decisões tomadas na organização como um todo; saber identificar oportunidades e } \\
\text { ameaças (Dulewicz \& Higgs, 2005). }\end{array}$ \\
\hline $\begin{array}{l}\text { Visão e } \\
\text { imaginação }\end{array}$ & $\begin{array}{l}\text { Ser imaginativo e criativo; ter visão clara da estratégia da organização e saber } \\
\text { estabelecer prioridades alinhadas com esta estratégia; prever o impacto da visão de } \\
\text { membros da equipe na implementação de decisões e nos negócios da organização } \\
\text { (Dulewicz \& Higgs, 2005). }\end{array}$ \\
\hline $\begin{array}{l}\text { Análise crítica e } \\
\text { julgamento }\end{array}$ & $\begin{array}{l}\text { Sondar os fatos; identificar vantagens, desvantagens e falhas em propostas que lhe são } \\
\text { feitas; julgar fatos com assertividade, considerando o impacto de suas suposições } \\
\text { (Dulewicz \& Higgs, 2005). }\end{array}$ \\
\hline Atitude & $\begin{array}{l}\text { Tendências de sentimento, crenças, comportamento perante determinadas situações, } \\
\text { pessoas, grupos, objetos e questões. Tem três elementos: afetivo, cognitivo e } \\
\text { comportamental (PMI, 2007). }\end{array}$ \\
\hline Liderança & $\begin{array}{l}\text { Elemento essencial do ambiente de projetos. É a capacidade de criar um ambiente de } \\
\text { trabalho de cooperação; influenciar a equipe e torná-la comprometida com os objetivos } \\
\text { do projeto (Thamhain, 2004). }\end{array}$ \\
\hline Comunicação & $\begin{array}{l}\text { Processo no qual as informações são trocadas entre pessoas usando um sistema comum } \\
\text { de símbolos, sinais e comportamentos (PMI, 2007). }\end{array}$ \\
\hline
\end{tabular}

Fonte: Elaboração própria com base em (Dulewicz \& Higgs, 2005; PMI, 2007; Thamhain, 2004)

Além do questionário eletrônico e das entrevistas, foi aplicado o teste de personalidade do MBTI nos quatorze profissionais que responderam o questionário e nos quatro profissionais que foram entrevistados. O teste utilizado para identificar o tipo de personalidade dos pesquisados foi desenvolvido pela organização não-governamental Projeto Inspiira que realiza testes vocacionais para alunos e profissionais (Projeto Inspiira, 2013). Este teste é desenvolvido com base na teoria das preferências individuais do MBTI (Rajapogal, 2008). A 
fim de aferir a confiabilidade dos dados obtidos na pesquisa foi efetuado o processo de triangulação dos dados (Martins \& Theóphilo, 2009): (1) dados obtidos nas entrevistas; (2) resultados dos questionários e (3) resultado da pesquisa bibliográfica.

\section{ANÁLISE DOS RESULTADOS}

O questionário eletrônico foi enviado para trinta profissionais de TI; quatorze retornaram. Quanto ao perfil das empresas onde os respondentes atuam: doze (86\%) são de empresas de grande porte; dois (14\%) em empresas de médio porte. Os setores de negócio abrangidos pela amostra coletada são: financeiro (bancos), telecomunicações, consultoria de tecnologia e desenvolvimento de software, indústria, siderurgia e varejo.

A maioria dos respondentes atua como líderes técnicos - quatro; o restante da amostra se distribui entre as funções de gerente de equipe, líder de equipe, consultor, analista de sistemas, analista funcional e analista de negócios $\mathrm{O}$ propósito da análise dos resultados era determinar quais das competências e habilidades foram consideradas as mais importantes no gerenciamento de projetos de TI na opinião dos profissionais pesquisados. A tabela 3 apresenta a competências e habilidades consideradas mais importantes na amostra coletada:

Tabela 3: Competências e habilidades consideradas mais importantes para gerente de projetos de TI

\begin{tabular}{|l|r|r|r|r|r|}
\hline & $\begin{array}{c}\text { Extremamente } \\
\text { importante }\end{array}$ & Importante & $\begin{array}{c}\text { Neutro } \\
\text { (mais ou } \\
\text { menos) }\end{array}$ & $\begin{array}{c}\text { Não- } \\
\text { importante }\end{array}$ & $\begin{array}{c}\text { Extremamente } \\
\text { não- } \\
\text { importante }\end{array}$ \\
\hline $\begin{array}{l}\text { Habilidade de se } \\
\text { comunicar em } \\
\text { múltiplos níveis }\end{array}$ & $79 \%$ & $14 \%$ & & $7 \%$ & \\
\hline Liderança & $71 \%$ & & & & \\
\hline Discernimento & $71 \%$ & $29 \%$ & & & \\
\hline $\begin{array}{l}\text { Perspectiva } \\
\text { estratégica }\end{array}$ & $71 \%$ & $14 \%$ & $14 \%$ & & \\
\hline $\begin{array}{l}\text { Habilidade de } \\
\text { comunicação oral }\end{array}$ & $57 \%$ & $43 \%$ & & & \\
\hline $\begin{array}{l}\text { Experiência } \\
\text { profissional }\end{array}$ & $57 \%$ & $21 \%$ & $21 \%$ & & \\
\hline Motivação & $57 \%$ & $36 \%$ & $7 \%$ & & \\
\hline
\end{tabular}

A habilidade de comunicar com múltiplos níveis e a liderança foram as competências mais consideradas como "extremamente importantes", convergindo com a pesquisa de Stevenson e Starkweather (2010). Nas entrevistas, esta habilidade foi considerada "muito importante" pelos quatro entrevistados. Conforme visto na tabela 3, das sete competências mais valores pelos respondentes, seis estão relacionadas com soft skills, em concordância com o resultado do trabalho de Müller e Turner (2007).

Diferentemente do que poderia se esperar de profissionais de tecnologia, competências relacionadas à comunicação, liderança e discernimento sejam apontadas como mais importantes em gestão de projetos. Contudo, resultado muito semelhante foi apresentado por Müller e Turner (2007). Este resultado é coerente com a função crítica que o gerente de projetos de TI tem de manter o envolvimento de stakeholders e membros da equipe. Um dos entrevistados 
afirma, inclusive, que "gerenciamento de projetos não é uma coisa técnica. Ele tem que ser muito mais interpessoal, e não técnico.".

Por outro lado, a competência de conhecimento técnico obteve apenas um voto como "extremamente importante"; $36 \%$ dos respondentes a consideraram "importante"; $14 \%$ "neutro", 29\% não-importante e 14\% “extremamente não-importante", concordando com os argumentos de Wateridge (1997) e Sumner et al. (2006) de que o conhecimento técnico não é mais o fato mais impactante para o sucesso de projetos de TI.

Por fim, apenas 36\% dos respondentes consideram a certificação de PMP essencial para o bom gerenciamento de projetos, enquanto 57\% consideram a certificação PMP sem relevância para garantia de eficiência no trabalho do gerente de projetos. A pesquisa demonstra que os pesquisados consideram muito mais relevante ao sucesso do projeto a experiência do gerente que sua formação acadêmica ou uma certificação de mercado. Um dos entrevistados afirmou que "as habilidades mais exigidas são as comportamentais que não se ensinam no meio acadêmico.". Por fim, foram analisados os resultados do testes de personalidade dos pesquisados. Dos trinta testes enviados, 17 foram respondidos. A figura X ilustra a distribuição dos tipos de personalidade na amostra da pesquisa:

Figura 3: Distribuição dos tipos de personalidade na amostra

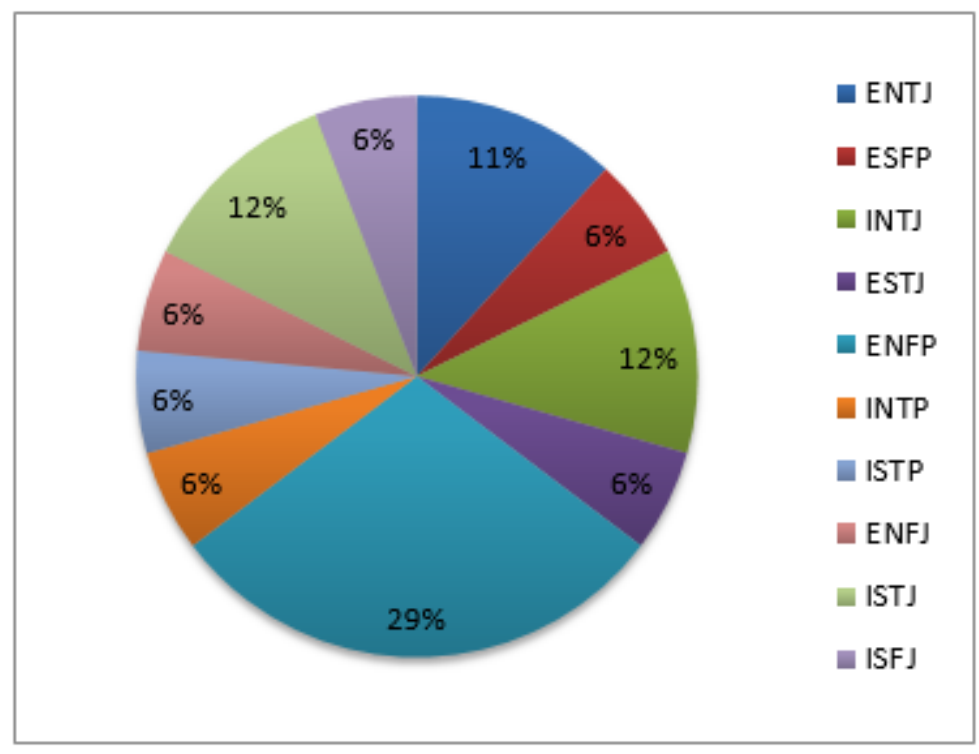

Os resultados dos testes de personalidade, das entrevistas e do questionário eletrônico foram cruzados, conforme mostra a tabela 4. Algumas competências e habilidades foram excluídas da análise, pois se tratavam de competências não se relacionavam com aspectos interpessoais e comportamentais. A partir do cruzamento de informações, foi possível identificar quais competências e habilidades eram compatíveis com o tipo de personalidade do pesquisado. Ademais, foi possível avaliar se o seu tipo de personalidade era ou não compatível com as competências que os entrevistados apontaram como prioritárias. A análise para identificação da relação entre os tipos de personalidade e os resultados do levantamento de dados foi baseada na pesquisa de Vargas (2005). Esta análise forneceu dados interessantes, como por exemplo, respondentes que classificaram liderança como "extremamente importante" cujo tipo de personalidade segundo MBTI tem personalidade mais introspectiva; ou, pesquisados que profissionais que identificam a habilidade de adaptação como essencial, mas que têm preferência por regras e estruturas mais estáticas. 
Tabela 4: Cruzamento do teste MBTI com a avaliação dos respondentes sobre as competências importantes para gerentes de projetos de TI

\begin{tabular}{|c|c|c|c|c|c|c|c|c|c|c|c|c|c|c|c|c|c|c|c|c|}
\hline Resp* & $\begin{array}{l}\text { Tipo } \\
\text { MBTI } \\
\end{array}$ & C1 & $\mathrm{C2}$ & $\mathrm{C3}$ & C4 & C5 & C6 & C7 & C8 & C9 & C10 & C11 & C12 & C13 & C14 & C15 & C16 & C17 & C18 & C19 \\
\hline R1 & ESTJ & 5 & 4 & 4 & 5 & 5 & 4 & 4 & 3 & 3 & 4 & 4 & 4 & 4 & 5 & 4 & 4 & 4 & 4 & 4 \\
\hline R2 & INTP & 5 & 4 & 4 & 4 & 4 & 4 & 5 & 3 & 3 & 4 & 2 & 3 & 5 & 4 & 4 & 4 & 5 & 3 & 4 \\
\hline R3 & ESFP & 5 & 3 & 5 & 5 & 5 & 5 & 5 & 5 & 5 & 3 & 3 & 5 & 3 & 5 & 3 & 3 & 3 & 3 & 5 \\
\hline R4 & INTJ & 5 & 4 & 5 & 5 & 5 & 5 & 4 & 3 & 5 & 5 & 3 & 5 & 3 & 3 & 5 & 5 & 3 & 4 & 5 \\
\hline R5 & ENFP & 5 & 5 & 5 & 5 & 5 & 5 & 5 & 5 & 5 & 5 & 5 & 5 & 5 & 5 & 5 & 5 & 5 & 5 & 5 \\
\hline R6 & ENFP & 5 & 5 & 3 & 5 & 4 & 4 & 5 & 4 & 4 & 4 & 5 & 4 & 4 & 3 & 4 & 4 & 4 & 4 & 5 \\
\hline R7 & ENFJ & 4 & 5 & 5 & 4 & 4 & 4 & 4 & 5 & 5 & 4 & 2 & 4 & 4 & 4 & 4 & 5 & 3 & 5 & 5 \\
\hline R8 & INTJ & 5 & 4 & 5 & 5 & 5 & 5 & 5 & 4 & 4 & 5 & 4 & 4 & 4 & 3 & 4 & 5 & 5 & 5 & 5 \\
\hline R9 & ENFP & 5 & 4 & 4 & 5 & 4 & 4 & 5 & 4 & 4 & 5 & 5 & 5 & 4 & 3 & 5 & 5 & 5 & 4 & 5 \\
\hline R10 & ENTJ & 5 & 5 & 5 & 5 & 5 & 5 & 5 & 4 & 5 & 4 & 4 & 5 & 2 & 5 & 4 & 5 & 5 & 4 & 4 \\
\hline R11 & ISFJ & 5 & 5 & 5 & 5 & 5 & 4 & 5 & 4 & 4 & 4 & 4 & 5 & 4 & 5 & 5 & 5 & 5 & 4 & 5 \\
\hline R12 & ENFP & 5 & 5 & 4 & 5 & 5 & 3 & 4 & 3 & 4 & 5 & 2 & 5 & 3 & 2 & 5 & 5 & 3 & 4 & 5 \\
\hline R13 & ISTJ & 5 & 4 & 3 & 5 & 4 & 5 & 4 & 5 & 4 & 4 & 4 & 5 & 5 & 4 & 5 & 4 & 5 & 4 & 4 \\
\hline R14 & ENFP & 5 & 5 & 4 & 5 & 5 & 5 & 5 & 4 & 3 & 5 & 3 & 5 & 4 & 3 & 4 & 5 & 5 & 3 & 4 \\
\hline R15 & ENTJ & 4 & 5 & 1 & 5 & 5 & 5 & 5 & 4 & 5 & 5 & 2 & 5 & 5 & 4 & 5 & 5 & 5 & 4 & 4 \\
\hline R16 & ISTJ & 5 & 4 & 4 & 4 & 4 & 5 & 5 & 4 & 4 & 4 & 3 & 4 & 4 & 4 & 4 & 3 & 5 & 5 & 5 \\
\hline
\end{tabular}

Nota: $*$ Resp $=$ respondente; $* *$ célula vermelha indica competência divergente das preferências do tipo de personalidade; *** célula verde indica competência concordante com preferência do tipo de personalidade $* * * *$ nome das colunas está na tabela 5 
Tabela 5: Relação do nome das colunas da tabela 4

\begin{tabular}{|c|c|c|c|c|c|c|c|}
\hline $\begin{array}{l}\text { Habilidade de se comunicar em múltiplos } \\
\text { níveis }\end{array}$ & C1 & Motivação & C6 & Intuição & C11 & \begin{tabular}{|l|}
$\begin{array}{l}\text { Habilidade de } \\
\text { realização }\end{array}$ \\
\end{tabular} & C16 \\
\hline $\begin{array}{l}\text { Habilidade de lidar com ambigüidade e } \\
\text { mudanças }\end{array}$ & $\mathbf{C 2}$ & Discernimento & C7 & Gerenciar recursos & C12 & Perspectiva estratégica & C17 \\
\hline Adaptação à cultura da empresa & C3 & Influência & C8 & $\begin{array}{l}\text { Habilidade de } \\
\text { persuadir }\end{array}$ & C13 & Visão e imaginação & C18 \\
\hline Liderança & $\mathbf{C 4}$ & Autoconsciência & C9 & Desenvolver recursos & C14 & $\begin{array}{l}\text { Análise crítica e } \\
\text { julgamento }\end{array}$ & C19 \\
\hline Habilidade de comunicação oral & C5 & $\begin{array}{l}\text { Resiliência } \\
\text { emocional }\end{array}$ & C10 & Delegar & C15 & & \\
\hline
\end{tabular}




\section{CONCLUSÃO}

A partir de pesquisa exploratória realizada à luz da literatura e com base em dados coletados entre profissionais de TI através de questionário eletrônico e entrevistas, somado ao cruzamento dos resultados do teste de personalidade do MBTI aplicado nos pesquisados, este trabalho cumpriu o objetivo de mostrar a importância dos soft skills no desenvolvimento das competências dos gerentes de projetos de TI, ao responder às seguintes questões (1) "qual o impacto dos soft skills no gerenciamento de projetos de TI?" e (2) "o tipo de personalidade do gerente de projetos pode influenciar no desenvolvimento de seus soft skills?".

A primeira questão de pesquisa foi respondida ao comprovar que profissionais de TI vêem os soft skills como competências essenciais em um gerente de projetos de TI. Das sete competências mais valorizadas pelos respondentes, seis eram relacionadas a soft skills. Liderança e comunicação foram citados como "skills fundamentais", pois o gerente de projetos é o responsável por influenciar e conduzir a equipe de projetos a fim de atingir um objetivo esperado. Assim como evidenciado por vários autores como Kerzer (2009), Wateridge (1998) e Sumner (2006) os conhecimento técnico não é tido como primordial para a formação de bom profissional em gestão de projetos. Para responder a segunda questão da pesquisa, foi realizado um cruzamento dos tipos de personalidade dos pesquisados e a classificação de importância que estes deram a determinadas competências em gerenciamento de projetos. A partir deste cruzamento, pois possível identificar que apesar de reconhecer a importância de uma determinada competência, o profissional tem um tipo de personalidade que pode dificultar o processo de desenvolvimento desta competência.

A principal limitação da pesquisa é a limitação de amostras. Devido à limitação de amostras, não foi possível, por exemplo, validar os argumentos de que determinados tipos de personalidade são mais adequados para gestão de projetos que outros (Cohen, Ornoy, \& Keren, 2013). Para estudos futuros uma amostra muito maior deverá ser coletada a fim de se obter um resultado mais abrangente e assertivo.

Todavia, esta pesquisa identifica interessantes possibilidades para estudos futuros para analisar: (1) a relação entre o tipo de personalidade e o desenvolvimento de habilidades interpessoais e comportamentais dos profissionais de TI; (2) soluções de questões críticas que têm afetado as relações sociais e de comunicação no gerenciamento de projetos de TI (Müller, 2003; Sumner et al., 2006; Wateridge, 1997); (3) o uso de testes de personalidade, como o MBTI, para auxiliar profissionais de TI no desenvolvimento de soft skills; (4) quais competências interpessoais e comportamentais são mais necessárias para empresas brasileiras do setor de TI.

O estudo das competências essenciais do gerente de projetos é sempre pertinente, pois a partir da pesquisa é possível ajudar profissionais a serem mais eficientes em suas funções, contribuindo para o sucesso das organizações em que atuam. Como Wateridge (1997) argumenta, o estudo das competências do gerente de projetos é como um quebra-cabeça infinito que vai se completando aos poucos. E "completar o quebra-cabeça" é um campo promissor para mundo acadêmico e científico. 


\section{REFERÊNCIAS BIBLIOGRÁFICAS}

Assis, C. B. (2011). Governança e gestão da tecnologia da informação: diferenças na aplicação em empresas brasileiras - dissertação de mestrado. Escola Politécnica da Universidade de São Paulo (Poli/USP).

Belzer, K. (2001). Project management: still more art than science. In PM Forum Featured Papers.

Cohen, Y., Ornoy, H., \& Keren, B. (2013). MBTI Personality Types of Project Managers and Their Success: A Field Survey. Project Management Journal, (June). doi:10.1002/pmj

Dalfovo, M. S., Lana, R. A., \& Silveira, A. (2008). Métodos quantitativos e qualitativos: um resgate teórico. Revista Interdisciplinar Científica Aplicada, 2(n. 4), 1-13.

Dolfi, J., \& Andrews, E. J. (2007). The subliminal characteristics of project managers: An exploratory study of optimism overcoming challenge in the project management work environment. International Journal of Project Management, 25(7), 674-682. doi:10.1016/j.ijproman.2007.02.002

Dulewicz, V., \& Higgs, M. (2005). Assessing leadership styles and organisational context. Journal of Managerial Psychology, 20(2), 105-123. doi:10.1108/02683940510579759

Fleury, M. T. L., \& Fleury, A. (2001). Construindo o Conceito de Competência. RAC, (Edição Especial), 183-196.

Gil, C. A. (2002). Como Elaborar Projetos de Pesquisa (4a. edição.). Editora Atlas.

Kerzner, H. (2009). Project Management: A Systems Approach to Planning, Schedulling and Controlling (10th editi.). New Jersey: John Wiley \& Sons, Inc.

Manfredi, S. M. (1998). Trabalho, qualificação e competência profissional - das dimensões conceituais e políticas. Educação \& Sociedade, 19(64). doi:10.1590/S010173301998000300002

Martins, G. de A., \& Theóphilo, C. R. (2009). Metodologia da investigação científica para ciências sociais aplicadas - 2a. edição (2nd ed.). São Paulo, SP, Brazil: Editora Atlas S.A.

Müller, R. (2003). Determinants for external communications of IT project managers. International Journal of Project Management, 21(5), 345-354. doi:10.1016/S02637863(02)00053-4

Müller, R., \& Turner, J. R. (2007). Matching the project manager's leadership style to project type. International Journal of Project Management, 25(1), 21-32. doi:10.1016/j.ijproman.2006.04.003

Pant, I., \& Baroudi, B. (2008). Project management education: The human skills imperative. International Journal of Project Management, 26(2), 124-128. doi:10.1016/j.ijproman.2007.05.010 
PMI. (2007). Project Manager Competency Development ( PMCD ) Framework (2nd ed.). Pennsylvania, USA: Project Management Institute, Inc.

PMI. (2013). A Guide to the Project Management Body of Knowledge ( PMBOK® Guide ) - Portuguese (5th ed.). Pennsylvania, USA: Project Management Institute, Inc.

Projeto Inspiira. (2013). [software]. Walnut, CA, USA: Projeto Inspiira.

Rabechini Jr., R. (2001). A importância das habilidades do gerente de projetos. Revista de Administração da Universidade de São Paulo, 36(1).

Rabechini Jr., R., \& Carvalho, M. M. De. (2003). Perfil das competências em equipes de projetos. RAE-eletrônica, 2, 1-17. Retrieved from http://www.rae.com.br/eletronica/index.cfm?FuseAction=Artigo \&ID=1333\&Secao=OPERA/ LOGI\&Volume $=2 \&$ Numero $=$

Rajapogal, N. (2008). Myers-Briggs type indicator(MBTI): Examining Behavioural aspects of executives in IT. Foundation for Organisational Research \& Education, 26(1).

Rao, M. S. (2012). Myths and truths about soft skills. $T+D, 66(5), 48$.

Ribeiro Filho, J. F., Lopes, J. E. de G., Pederneiras, M. M. M., Almeira, L. B. de, \& Ribeiro, M. T. J. de B. (2010). Características da personalidade de estudantes de ciências contábeis: Análise do conhecimento baseado no Modelo Myers-Briggs Type Indicator (MBTI). Contabilidade, Gestão e Governança, 13(2).

Shenhar, A. J., \& Wideman, R. M. (2002). Optimizing sucess by matching management style to project type. PMForum Site.

Smith, L. W. (2001). The effects of project manager personality profiles on projects. Nashiville, Tenessee, USA.

Stevenson, D. H., \& Starkweather, J. A. (2010). PM critical competency index: IT execs prefer soft skills. International Journal of Project Management, 28(7), 663-671. doi:10.1016/j.ijproman.2009.11.008

Sumner, M., Bock, D., \& Giamartino, G. (2006). Exploring the linkage between the characteristics of IT project leaders and project sucess. Information Systems Management, 23(4), 43.

Thamhain, H. J. (2004). Team leadership effectiveness in technology-based project environments. Project Management Journal, (December), 35-47.

Turner, J. R., \& Müller, R. (2005). The project manager's leadership style as a success factor on projects: a literature review. Project management journal, 36(2), 49-61. 
Vargas, R. V. (2005). Avoiding mistakes during the team acquisition: find the right people to the right function using MBTI. In PMI Global Congress Proceedings (pp. 1-9). Edinburgh, Scotland.

Wateridge, J. (1997). Training for IS/IT project managers: a way forward. International Journal of Project Management, 15(5), 283-288.

Wateridge, J. (1998). How can IS/IT projects be measured for success? International Journal of Project Management, 16(1), 59-63. doi:10.1016/S0263-7863(97)00022-7

Wideman, R. M. (1998). Dominant Personality Traits Suited to Running Projects Successfully (And What Type are You?). AEW Services.

Wideman, R. M. (2008). Project teamwork, personality profiles and the population at large: do we have enough of the right kind of people? Long Beach, California: Project Management Institute, Inc.

Zemke, R. (1992). Second thoughts about the MBTI. Training, 29(4), 43. 


\section{APÊNDICE A - QUESTINÁRIO UTILIZADO NO SURVEY E NAS ENTREVISTAS}

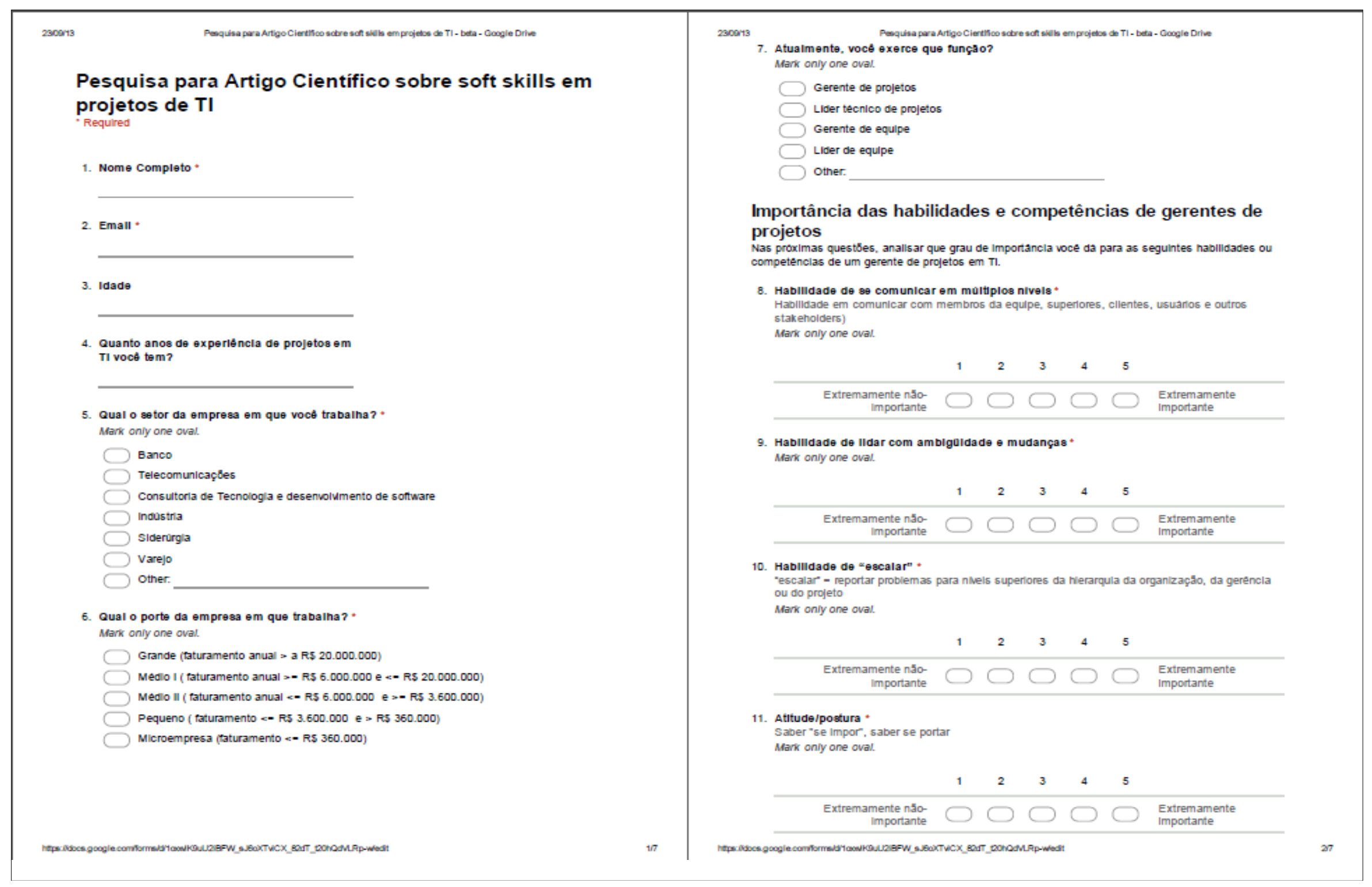

International Journal of Professional Business Review (JBReview), São Paulo V.1 N.1 2016, pp. 40-59, Jan/Jun 


\section{APÊNDICE A - QUESTINÁRIO UTILIZADO NO SURVEY E NAS ENTREVISTAS (cont)}

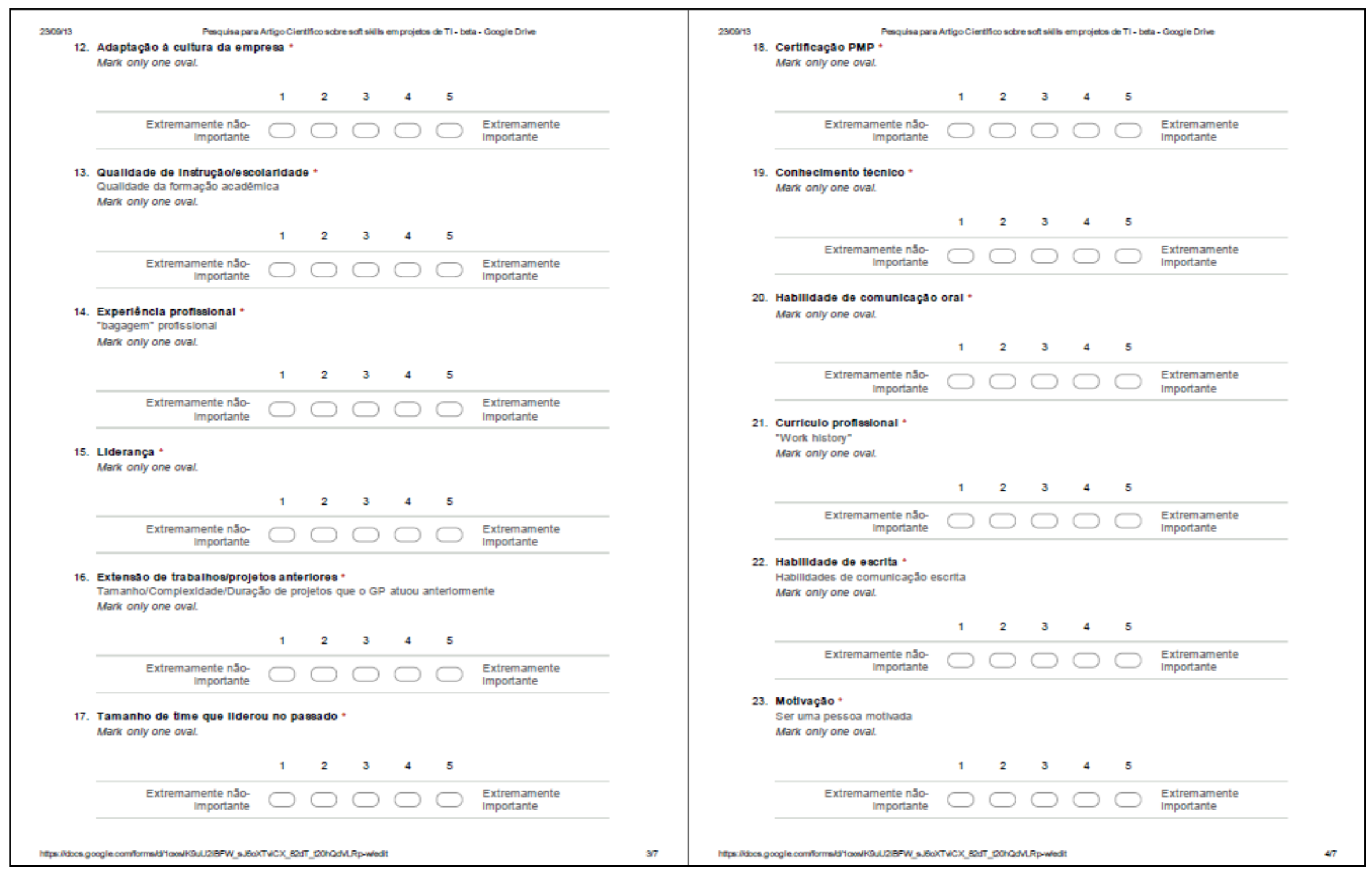

International Journal of Professional Business Review (JBReview), São Paulo V.1 N.1 2016, pp. 40-59, Jan/Jun 


\section{APÊNDICE A - QUESTINÁRIO UTILIZADO NO SURVEY E NAS ENTREVISTAS}

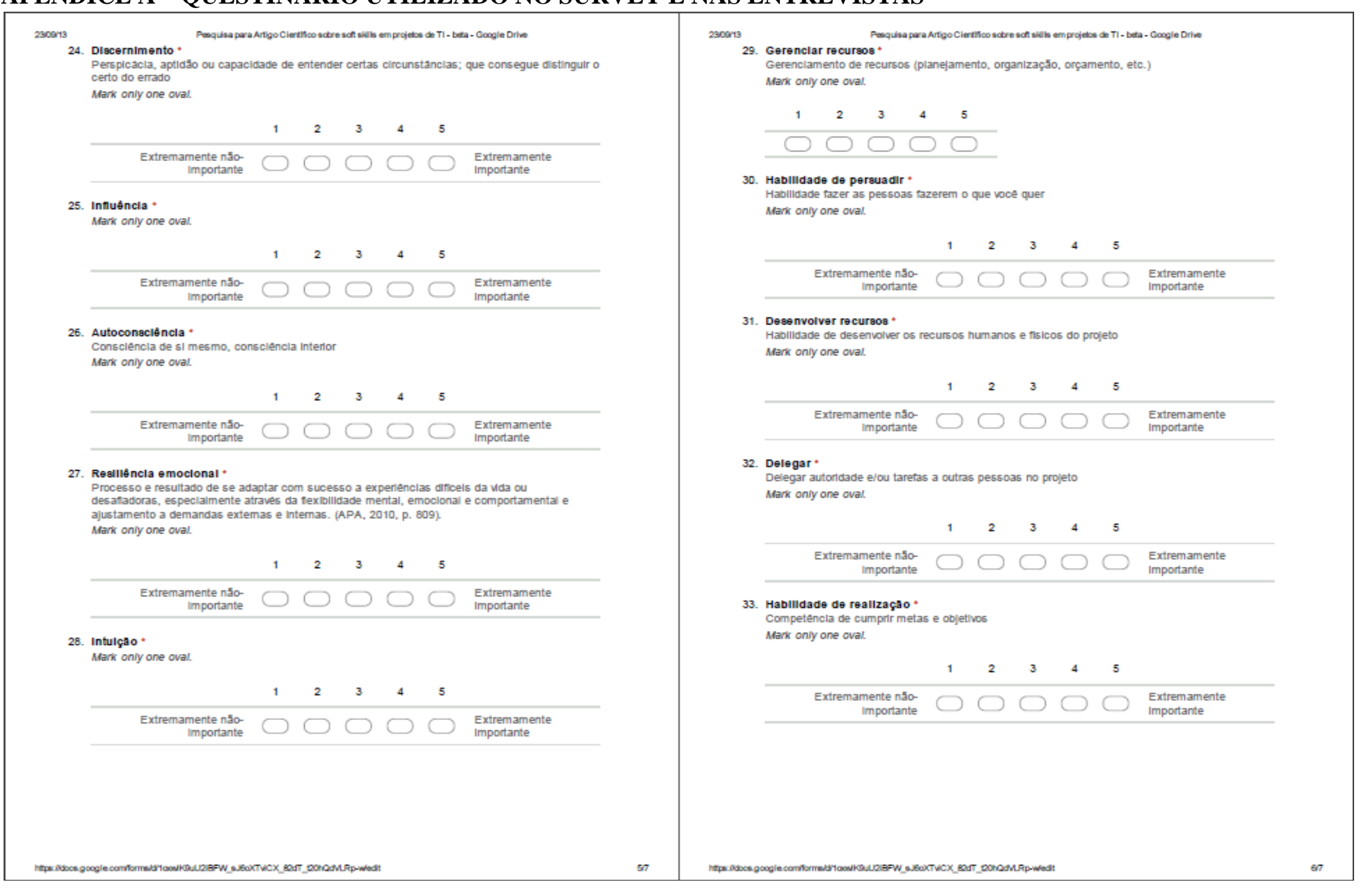

International Journal of Professional Business Review (JBReview), São Paulo V.1 N.1 2016, pp. 40-59, Jan/Jun 